\title{
Correlation of cord blood telomere length with birth weight
}

\author{
Siew-Peng Lee ${ }^{1}$, Prakash Hande ${ }^{2}$, George SH Yeo ${ }^{3}$ and Ene-Choo Tan ${ }^{1,4^{*}}$ (D)
}

\begin{abstract}
Background: Intrauterine growth restriction affects 3\% of newborns; and the lightest 10\% of whom are classified as small for gestational age (SGA). These low-birth weight newborns are at increased risk of neonatal morbidity such as hypoxia and hypoglycaemia. In later life, they are at higher risk of several age-related diseases such as cardiovascular and metabolic disorders and dementia. As having short telomeres is also associated with these diseases, we tested if these newborns might already start with shorter telomeres at birth.

Findings: Relative telomere lengths were determined using quantitative real-time PCR in cord blood samples from 195 newborns of Chinese ancestry. Based on the telomere length normalised to a single copy gene and a reference DNA sample as internal control, we found statistically significant correlations between relative telomere length and both unadjusted and gestational age-adjusted birth weight, with the lighter newborns having shorter telomeres. The SGA birth weight group comprising the bottom $10 \%$ of the samples also had the shortest telomeres compared to the medium and heaviest birth weight groups.
\end{abstract}

Conclusions: Our results indicate that there is reduction of cord blood telomere length for newborns with lower birth weight.

Keywords: Birth weight, Newborns, Quantitative PCR, Telomere length

\section{Background}

Telomeres are highly conserved hexamer repeats (TTAGGG) at the ends of all chromosomes [1]. They maintain chromosome stability by preventing the ends from fusing to each other and allow DNA replication at chromosome ends. Telomeres are longest at birth, with the size reported to range from about 7 to $20 \mathrm{~kb}[2,3]$. The number of hexamer repeats is reduced with each round of replication, leading to its shortening from birth until end of life in somatic tissues.

The enzyme telomerase which maintains and restores the terminal regions is only expressed in embryonic/ foetal cells, germ cells and other proliferating organs in adults. Short telomeres are associated with genome instability, cellular aging, some types of cancer, and several age-related diseases such as heart disease, atherosclerosis

\footnotetext{
*Correspondence: tanec@bigfoot.com

1 Research Laboratory, KK Women's and Children's Hospital, 100 Bukit Timah Road, Singapore, Singapore

Full list of author information is available at the end of the article
}

and diabetes $[4,5]$. Although the rate of telomere shortening is influenced by physiological ageing and environmental factors as well, there is good evidence of genetic contribution to telomere length $[6,7]$.

Several methods have been developed to assess telomere length. They include procedures which quantify the mean from total DNA extracted from a population of cells, and those which measure the telomere of individual chromosomes in a cell [8]. The most established method uses southern blot hybridization analysis of terminal restriction fragments (TRF) to measure the absolute telomere length and the subtelomeric regions. However, this method is labour-intensive and time-consuming, and requires large amount of input of starting material (0.5-5 $\mu \mathrm{g}$ DNA).

With the advent of real-time polymerase chain reaction platforms, a method based on quantitative real-time PCR (qPCR) which requires about $100 \times$ less DNA, has higher throughput and faster turnaround time has become more commonly used [9]. The measurements are also more representative of telomere length as telomere primers 
specifically hybridise to human telomere sequences and do not depend on restriction enzyme recognition sites outside of the telomeric region which might lead to additional non-telomeric sequences being counted [10].

In this study, we tested the hypothesis that cord blood telomere length might be directly related to birth weight. Our objective was to determine the relationship between birth weight and cord blood telomere length in newborns of Chinese descent that were born in our hospital. We measured the telomere length of these cord blood samples using qPCR to assess correlation with birth weight, taking into account gestational age and maternal age.

\section{Methods}

\section{Study samples}

The study protocol was approved by the SingHealth Institutional Review Board which oversees all research activities in the hospital. Inclusion criteria were deliveries from mothers of Chinese descent from singleton pregnancies with no recognizable congenital disorders. Approval was granted for the anonymous collection of cord blood and limited demographic data without requiring written informed consent.

Cord blood samples were collected between March 2011 and April 2012 in our hospital, for cases that met inclusion criteria and were not involved in other research studies requiring cord blood, had not consented to donate to cord blood bank, or had not planned to store it with a private operator. Data on gestational age, gender and birth weight of the newborns, and maternal age were anonymously collected.

\section{Experimental details}

Genomic DNA was extracted from the cord blood of 195 Chinese newborns (98 males, 97 females) using Puregene Blood Kit (Qiagen GmbH, Hilden, Germany). The quantity and quality of extracted DNA was determined by using a NanoDrop 1000 spectrophotometer (Fisher, Wilmington, DE, USA).

Telomere length assessment was done by qPCR, with $36 B 4$ as the reference gene for normalisation using published primer sequences [9]. Amplification for both telomere copy number and the reference gene was done in separate wells but in the same experiment under the same PCR cycling conditions to reduce inter-experimental variation, in batches of five samples per experiment. The reaction mixture for $36 \mathrm{~B} 4$ was made up of $15 \mathrm{ng}$ genomic DNA, $1 \times$ Roche FastStart Universal SYBR Green Master (ROX) (Roche Diagnostics Deutschland GmbH, Mannheim, Germany), $300 \mathrm{nM}$ of the forward and $500 \mathrm{nM}$ of the reverse primers while the telomere reaction mix had $270 \mathrm{nM}$ of the forward and $900 \mathrm{nM}$ of the reverse primers. Water was added to a final volume of $20 \mu \mathrm{l}$. DNA samples from two healthy females were included in every experiment as internal calibration to control for interexperimental variability. Amplification for each sample was done in quadruplicates in the Applied Biosystems StepOnePlus real time PCR system (Applied Biosystems Incorporated, Foster City, CA, USA) using the following cycling condition: $50{ }^{\circ} \mathrm{C}$ for $2 \mathrm{~min}$ and $95^{\circ} \mathrm{C}$ for $10 \mathrm{~min}$, followed by 40 cycles of denaturation at $95{ }^{\circ} \mathrm{C}$ for $15 \mathrm{~s}$, and annealing-extension at $56{ }^{\circ} \mathrm{C}$ for $1 \mathrm{~min}$ and dissociation curve analysis.

Result was analysed using the Applied Biosystems StepOne $^{\mathrm{TM}}$ software (version 2.1). $\mathrm{C}_{\mathrm{T}}$ values were obtained by setting the threshold at the exponential phase of amplification. The average $C_{T}$ values of the best three of the quadruplicates were used with one set of data discarded. Telomere copy number relative to single copy gene (T/S) ratio was calculated from the $\mathrm{C}_{\mathrm{T}}$ values using the follow-

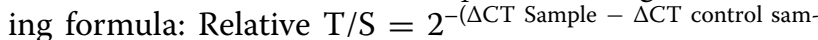
${ }^{\text {ple })}=2^{-(\Delta \Delta C T)}$. Where $\Delta C_{T}^{\text {Sample }}=$ Sample $C_{T(\text { tel })}-$ Sample $\mathrm{C}_{\mathrm{T}(36 \mathrm{~B} 4)}$ and $\Delta \mathrm{C}_{\mathrm{T}}^{\text {control }}=$ Control $\mathrm{C}_{\mathrm{T}(\text { tel })}-$ Control $\mathrm{C}_{\mathrm{T}(36 \mathrm{~B} 4)}$. Relative telomere length (RTL) was then obtained by comparing the $\mathrm{T} / \mathrm{S}$ ratio of the test sample relative to an internal control DNA sample included in every experiment to correct for any batch variation for assays done at different times. The coefficient of variation for RTL values obtained using Control \#1 is $12.7 \%$ and for Control \#2 is $12.2 \%$.

\section{Statistical analysis}

All analyses were carried out using IBM Statistical Package for the Social Sciences version 19 (SPSS Inc., Chicago, USA). A $P$ value of $<0.05$ was considered as statistically significant. Quantitative variables are expressed as mean and standard deviation. For each sample, RTL used was the average of the best three of the four replicates relative to each of the two control DNA samples, and gestational age-adjusted birth weight was generated by dividing the birth weight by gestational age. Correlation between individual quantitative variables was evaluated using a Pearson test. The relationship between RTL and birth weight was also explored while controlling for maternal age using partial correlation.

The newborns were placed into three groups based on clinical definition that is generally used to categorise extreme fetal growth groups (MSD Manual: http://www. $\mathrm{msdmanuals.com/professional/pediatrics/perinatal-}$ problems). Age-adjusted birth weight was used to classify the samples: lightest $10 \%(\mathrm{n}=20)$ in the small for gestational age group (SGA), heaviest $10 \%(n=20)$ in the large for gestational age group (LGA), and the rest $(n=155)$ in the appropriate for gestational age group (AGA). Differences between the three groups were assessed using ANOVA for quantitative variables and Chi Square for categorical variables. 


\section{Results}

A total of 235 samples were collected from January 2011 to March 2012. Birth weight ranged from 610 to $5184 \mathrm{~g}$ (mean $3186.1 \pm 492.4 \mathrm{~g}$ ) and gestational age from 32 to 41 weeks (mean $38.15 \pm 1.23$ weeks). Of the 195 samples with DNA available for telomere length qPCR analysis, there were 97 males and 98 females. The experimentally determined telomere length showed normal distribution (Table 1). There was no statistically significant difference for all variables between male and female samples (Table 2).

Correlations between RTL and study variables were presented in Table 3. RTL values obtained using Control DNA \#1 had lower standard deviation (0.007 versus 0.012 for Control DNA \#2) and standard error of mean (0.00604 versus 0.00784) compared to Control DNA \#2. There was highly significant correlation between the RTL from Control DNA \#1 and that from Control DNA \#2 (Table 3). When measured against a single copy gene, the longest RTL was almost $2 \times$ more than the shortest when calibrated against both Control DNA \#1 (range 0.54451.0860; SD: 0.1007) and Control DNA \#2 (range 0.73511.5210; SD: 0.1261).

There was a statistically significant correlation of RTL with birth weight, with the heavier newborns having longer RTL (Fig. 1; Table 3). There was also correlation of RTL with TRF data obtained previously [3] for these samples (Table 3). However, there was no statistically significant correlation with maternal age and gestational age. Adjusting for maternal age did not result in any difference in statistical significance.

Analysis using tertile birth weight groups and different models (linear, quadratic, cubic, polynomial) did not reveal any statistically significant correlation. The extreme group design was then used to classify the newborns into three groups based on age-adjusted birth weight: lightest 10\% (Group SGA), heaviest 10\% (Group LGA), and the rest (Group AGA). There were statistically

Table 1 Characteristics of the cord blood samples analyzed

\begin{tabular}{lclll}
\hline & Mean (SD) & Median & Minimum & Maximum \\
\hline Mother's age in years & $32.7(4.9)$ & 33.0 & 17 & 47 \\
Birth weight in grams $^{\mathrm{a}}$ & $3235(501)$ & 3162 & 1128 & 5184 \\
$\begin{array}{l}\text { Gestational age in } \\
\text { weeks }^{\mathrm{a}}\end{array}$ & $38.2(1.1)$ & 38.0 & 32 & 41 \\
qPCR RTF (Control \#1) & $0.996(0.126)$ & 0.991 & 0.735 & 1.521 \\
qPCR RTF (Control \#2) & $0.826(0.101)$ & 0.823 & 0.545 & 1.09 \\
TRF (kb) & $12.7(2.5)$ & 12.6 & 0.663 & 19.2 \\
\hline
\end{tabular}

a Kolmogorov-Smirnov test of normality indicates significant deviation from normal distribution
Table 2 Comparison of characteristics between male and female samples

\begin{tabular}{lclll}
\hline & \multicolumn{1}{l}{ Mean (SD) } & F & P \\
\cline { 2 - 3 } & Males (n=97) & Females (n= 98) & & \\
\hline Mother's age (years) & $32.7(4.9)$ & $32.6(5.2)$ & 0.015 & 0.902 \\
Birth weight (grams) & $3235(501)$ & $3158(483)$ & 1.204 & 0.274 \\
Gestational age & $38.2(1.1)$ & $38.15(1.23)$ & 0.248 & 0.619 \\
$\quad$ (weeks) & & & & \\
qPCR RTF/Control \#1 & $0.989(0.501)$ & $1.003(0.127)$ & 0.551 & 0.459 \\
qPCR RTF/Control \#2 & $0.821(0.101)$ & $0.831(0.101)$ & 0.423 & 0.516 \\
TRF (kb) & $12.4(2.6)$ & $12.9(2.4)$ & 1.617 & 0.205 \\
\hline
\end{tabular}

a Data only available for 81 males and 78 females

significant differences among the groups in terms of RTL $(P=0.022)$ normalised to Control \#1 (Fig. 2a). Group SGA had a mean RTL of 0.7922 compared to 0.8537 for Group LGA $(P=0.037)$, with the mean for Group AGA at $0.8270(P=0.050)$. The difference between Group AGA and Group LGA was not statistically significant.

There was a statistically significant difference $(P=0.009)$ in mean gestational age for the three groups: with the mean for SGA at $37.30 \pm 2.27$ weeks, AGA at $38.24 \pm 1.03$ and LGA at $38.30 \pm 0.865$. The median for all three groups were the same at 38.00 weeks. There was no statistically significant difference between the three groups in the mean for maternal age $(P=0.653)$ or TRF measured previously $(P=0.332)$.

Eight cases were from preterm deliveries of between 32 and 36 weeks (mean gestational age $34.6 \pm 1.69$ weeks). Although the preterm group has shorter RTL of $0.933 \pm 0.139$ compared to the $0.998 \pm 0.125$ for the full-term group (gestational age $\geq 36$ weeks; mean $38.3 \pm 0.952$ weeks), the difference was not statistically significant $(P=0.153)$. There was also no statistically significant difference in the mean maternal age between the preterm and full-term birth groups $(P=0.911)$.

Table 3 Correlation analysis of RTL with different variables

\begin{tabular}{|c|c|c|c|c|}
\hline & \multicolumn{2}{|c|}{ RTL (Control \# 1) } & \multicolumn{2}{|c|}{ RTL (Control \#2) } \\
\hline & Pearson's R & $P$ (2-tailed) & Pearson's R & $P($ 2-tailed $)$ \\
\hline TRF & 0.144 & 0.069 & 0.195 & $0.014^{*}$ \\
\hline Maternal age & 0.001 & 0.989 & -0.003 & 0.964 \\
\hline Gestational age & 0.108 & 0.131 & 0.012 & 0.863 \\
\hline $\begin{array}{l}\text { Birth weight } \\
\text { (BW) }\end{array}$ & 0.201 & $0.005^{*}$ & 0.158 & $0.028^{*}$ \\
\hline Age-adjusted BW & 0.199 & $0.005^{*}$ & 0.172 & $0.017^{*}$ \\
\hline $\begin{array}{l}\text { Control DNA \#2 } \\
\text { (or \#1) }\end{array}$ & 0.801 & $0.000^{*}$ & 0.801 & $0.000^{*}$ \\
\hline
\end{tabular}

* Statistically significant 

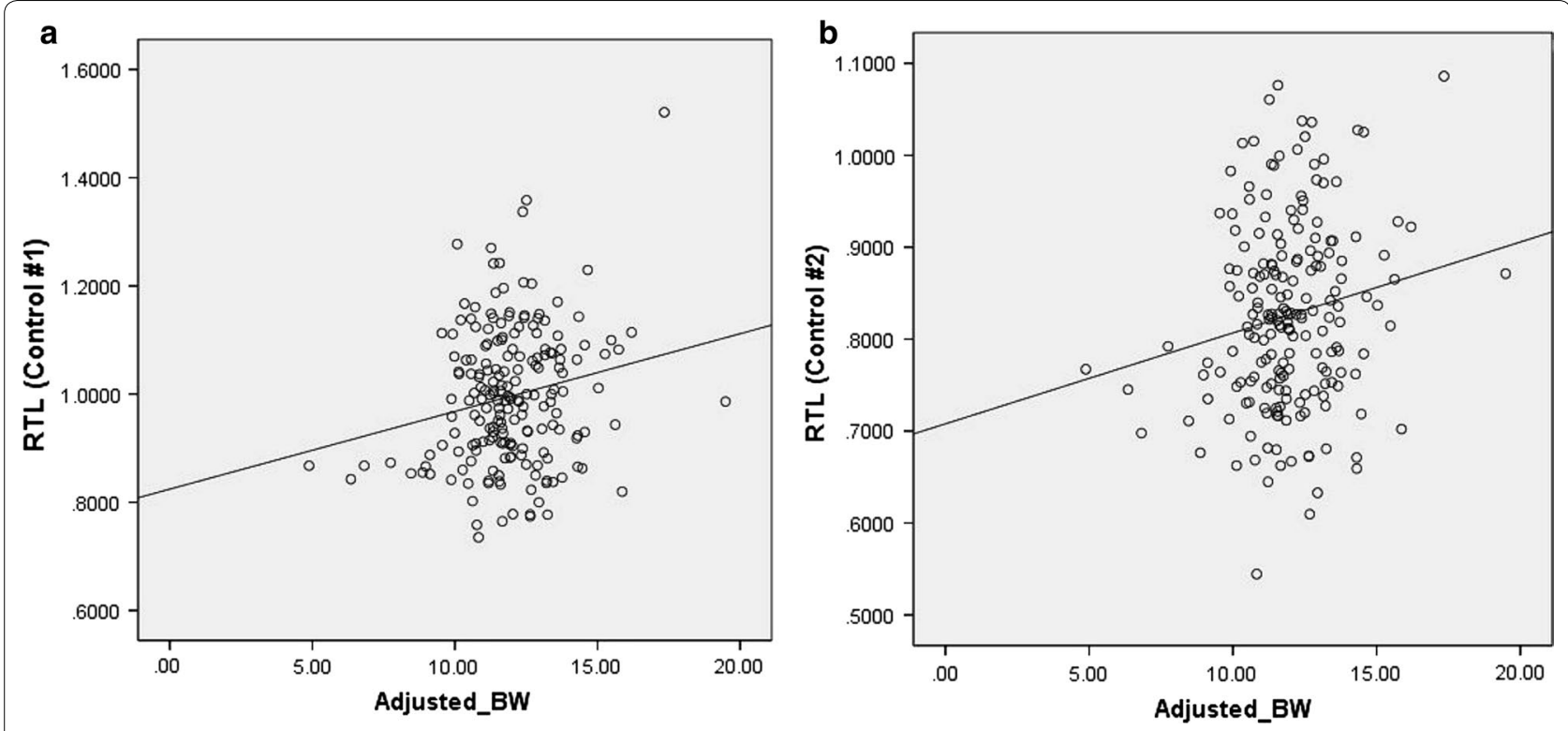

Fig. 1 Correlation of RTL with age-adjusted birth weight $\mathbf{a}$ using Control \#1 (Pearson correlation $=0.198, P=0.005$ ) and $\mathbf{b}$ using Control \#2 (Pearson correlation $=0.171, P=0.017$ )

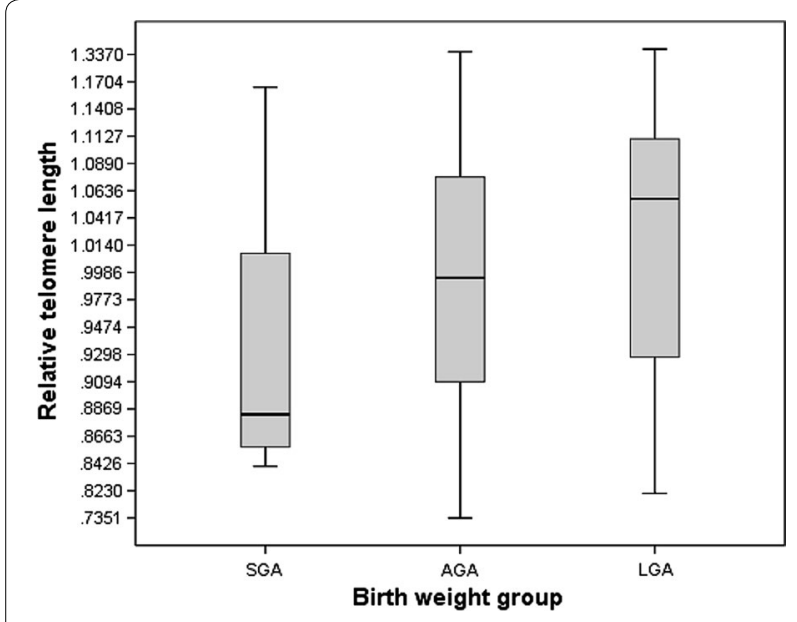

Fig. 2 Mean RTL for the three birth weight groups based on clinical definition of birth weight (SGA/AGA/LGA) using results from Control $\# 1(P=0.022)$

\section{Discussion}

It has been shown that the DNA extraction method used can affect telomere length measurement due to differences in purity and integrity $[11,12]$. In this study, all the samples were extracted using the same method. The RTL results are largely consistent between experiments and also between different reference control DNA samples.

Telomere length in this study cohort had been determined by TRF previously [3], and was measured by qPCR as a comparison in the current study. Similar to other recent studies $[13,14]$, there was only moderate correlation between telomere length measured by qPCR and TRF. Our correlation appears to be lower than some of the published data. One explanation is that our population on the whole was more polymorphic at the subtelomeric region restriction enzyme digestion sites used in TRF analysis, which might have resulted in higher values for telomere length for some samples $[15,16]$.

Our result of shorter telomeres in lower birth weight newborns is consistent with the reported reduced telomere length in intrauterine growth restriction (IUGR) placentas compared to controls as measured by quantitative FISH $[17,18]$. Our sample size is larger than most previous studies, with all the samples obtained from a single ethnic group. Our data showed direct correlation of both unadjusted and gestational age-adjusted birth weight with relative telomere length.

Additional analysis using tertile birth weight groups and different models did not reveal any statistically significant correlation. Conversely, there is a statistically significant difference when the newborns are divided into three groups based on gestational age-adjusted birth weight, with the shortest telomeres found in the group with the lowest birth weight. This extreme group method of categorizing birth weight (SGA/AGA/LGA) is typically used by studies investigating the association of telomere length with birth parameters. It is based on clinical relevance as both LGA and SGA groups are at higher risk of maternal health complications, adverse neonatal outcomes and future health morbidity. LGA newborns are 
at increased risk of birth injuries during deliveries. They may have polycythemia, hypoglycemia and immature lungs if the mothers are diabetic, and increased risk of diabetes and obesity in adulthood [19]. However, the risk for these later life disorders could also be due to genetic predisposition if their mothers were predisposed to gestational diabetes during the pregnancies.

For SGA neonates and in particular, those with intrauterine growth restriction (IUGR), there is an increased risk of cardiovascular and metabolic diseases [20]. Some of the diseases are also those which have been reported to be associated with short telomeres. Shorter cord blood telomere length has also been associated with perinatal outcomes such as gestational diabetes and preterm premature rupture of membranes [21, 22]. The risk for those born as SGA might be related to being exposed to unfavorable in utero conditions that lead to oxidative stress and high turnover of cells during the embryonic period, both of which are known to lead to reduced telomere length. The unfavorable growth might also predispose them to the development of adult diseases such as hypertension, cardiovascular disease, and type 2 diabetes [23]. As some IUGR newborns are known to have conditions linked to aged phenotypes such as chronic hypertension and hyperglycemia, it is not implausible that their telomeres might also showed some "ageing" and appear shorter than age-matched controls with normal birth weight. Experimental evidence from a study subjecting lymphocyte cultures derived from cord blood samples to a genotoxic agent showed that those with shorter telomeres had more genetic damage as measured by the frequency of binucleated cells with micronuclei [24].

A recent study $(\mathrm{N}=69)$ found an inverse correlation between telomere length and birth weight groups, with the LGA group having the shortest telomeres and the SGA group having the longest compared to their average weight counterparts [25]. In our study cohort, telomere length for the top $10 \%$ in terms of age-adjusted birth weight was longer and not shorter than the other two groups, while the bottom $10 \%$ had the shortest telomeres. Our results are in agreement with two other recent studies ( $\mathrm{N}=54$ and 103) using $\mathrm{qPCR}$ on cord blood DNA, both reporting that telomere length at birth was longer in larger newborns [26, 27]. Another study $(\mathrm{N}=66)$ found no association with birth weight but the data on telomere length for specific birth weight categories was not presented [28]. Similar to our data, they did not find statistically significant correlation between gestational age and telomere length. Using a different design, a group reported significant positive correlation between intra-pair differences of birth weight and telomere length for monozygotic but not dizygotic twin pairs, and that monozygotic twins with the larger difference in birth weight also have larger difference in telomere length [29]. These results suggest that the in utero environment which affect foetal growth also influences telomere length, and that TL is partly determined by the genetic background as the correlation is only statistically significant in monozygotic twin pairs. Inter-individual genetic variation could explain why some studies (especially those with small samples sizes) did not show correlation with birth weight, as the genetic factors influencing telomere length are largely unknown and thus could not be measured at present.

We previously reported that females have longer telomeres as measured by TRF. The data for qPCR RTL showed a similar trend although the difference was not statistically significant (Table 2). Recently, Wojcicki et al. also reported shorter telomere length at birth for males among Latino infants [27].

There are several limitations of this study. We do not have the clinical data on the mothers who might have medical conditions that impacted the growth of their foetuses; our data on birth weight and gestational age was collected anonymously and the mothers' pre-pregnancy weight or body mass index was not available for analysis. We also did not have the DNA from the parents for parental RTL measurement for correlation or adjustment of the newborns' RTL data. In addition, the qPCR method measures the average TL of all chromosomes in multiple cells, which cannot distinguish whether some cells or chromosomes have some telomeres that are significantly shorter or longer than the rest.

In summary, our RTL results using a medium sized cohort from a single ethnic group show a direct correlation of birth weight with mean qPCR telomere length which measures the true length of the telomere hexamers. As telomere length is a reflection of replicative history, the shorter telomeres could be a reflection of higher cellular turnover during the embryonic period for the newborns that had suboptimal growth. Future work could look into whether there might be a subpopulation of cells with shorter telomeres. The presence of individual chromosomes with much shorter telomeres can be explored using more sensitive methods that directly measure telomere length at the resolution of a single chromosome end.

\section{Abbreviations \\ AGA: appropriate for gestational age; FISH: fluorescent in situ hybridization; IUGR: intrauterine growth restriction; LGA: large for gestational age; qPCR: quantitative polymerase chain reaction; RTL: relative telomere length; SGA: small for gestational age; TRF: terminal restriction fragments.}

\section{Authors' contributions}

SPL performed the experiments, MPH, GSY and ECT designed the study, ECT obtained the funding, designed the study and analyzed the data, SPL and ECT drafted the manuscript. All authors read and approved the final manuscript. 


\begin{abstract}
Author details
${ }^{1}$ Research Laboratory, KK Women's and Children's Hospital, 100 Bukit Timah Road, Singapore, Singapore. ${ }^{2}$ Department of Physiology, Yong Loo Lin School of Medicine, National University of Singapore, Singapore, Singapore. ${ }^{3}$ Maternal-Fetal Medicine, KK Women's and Children's Hospital, Singapore, Singapore. ${ }^{4}$ Paediatrics Academic Clinical Programme, SingHealth Duke-NUS Medical School, Singapore, Singapore.
\end{abstract}

\section{Acknowledgements}

We thank Zaheedah Yahya and Shi-Ni Lim for assistance with generating the TRF data, and Wai-Yee Lim for consultation on statistical analysis.

\section{Competing interests}

The authors declare that they have no competing interests.

\section{Availability of data and materials}

The qPCR datasets analysed during the current study are available from the corresponding author on request.

\section{Consent for publication}

Ethics approval was granted for sample to be collected anonymously without informed consent. Publication of results was in accordance with institutional guidelines. All authors reviewed and approved the manuscript.

\section{Ethics approval and consent to participate}

Approval to conduct the study was granted by the SingHealth Institutiona Review Board for anonymous collection of cord blood samples without written informed consent.

\section{Funding}

This study was supported by Grants NMRC/EDG09may016 and NMRC/ CG/006/2013 from the National Medical Research Council, Ministry of Health, Republic of Singapore.

\section{Publisher's Note}

Springer Nature remains neutral with regard to jurisdictional claims in published maps and institutional affiliations.

Received: 8 August 2016 Accepted: 31 August 2017

Published online: 08 September 2017

\section{References}

1. Moyzis RK, Buckingham JM, Cram LS, Dani M, Deaven LL, Jones MD, Meyne J, Ratliff RL, Wu JR. A highly conserved repetitive DNA sequence, (TTAGGG)n, present at the telomeres of human chromosomes. Proc Natl Acad Sci USA. 1988;85:6622-6.

2. Friedrich $U$, Schwab M, Griese EU, Fritz P, Klotz U. Telomeres in neonates: new insights in fetal hematopoiesis. Pediatr Res. 2001;49:252-6.

3. Lim SN, Yahya Z, Zeegers D, Moe T, Kyaw EEP, Yeo GSH, Tan EC. Distribution of telomere length in the cord blood of Chinese newborns. Br J Med Med Res. 2013;3:1004-14.

4. D'Mello MJ, Ross SA, Briel M, Anand SS, Gerstein H, Pare G. Association between shortened leukocyte telomere length and cardiometabolic outcomes: systematic review and meta-analysis. Circ Cardiovasc Genet. 2015;8:82-90

5. Haycock PC, Heydon EE, Kaptoge S, Butterworth AS, Thompson A, Willeit P. Leucocyte telomere length and risk of cardiovascular disease: systematic review and meta-analysis. BMJ. 2014;349:94227.

6. Hjelmborg JB, Dalgard C, Moller S, Steenstrup T, Kimura M, Christensen K, Kyvik KO, Aviv A. The heritability of leucocyte telomere length dynamics. J Med Genet. 2015;52:297-302.

7. Honig LS, Kang MS, Cheng R, Eckfeldt JH, Thyagarajan B, LeiendeckerFoster C, Province MA, Sanders JL, Perls T, Christensen K, et al. Heritability of telomere length in a study of long-lived families. Neurobiol Aging. 2015;36:2785-90.
8. Turner KJ, Vasu V, Greenall J, Griffin DK. Telomere length analysis and preterm infant health: the importance of assay design in the search for novel biomarkers. Biomark Med. 2014;8:485-98.

9. Cawthon RM. Telomere measurement by quantitative PCR. Nucleic Acids Res. 2002;30:e47.

10. Aubert G, Hills M, Lansdorp PM. Telomere length measurement-caveats and a critical assessment of the available technologies and tools. Mutat Res. 2012;730:59-67.

11. Denham J, Marques FZ, Charchar FJ. Leukocyte telomere length variation due to DNA extraction method. BMC Res Notes. 2014;7:877.

12. Tolios A, Teupser D, Holdt LM. Preanalytical conditions and DNA isolation methods affect telomere length quantification in whole blood. PLoS ONE. 2015;10:e0143889.

13. Elbers CC, Garcia ME, Kimura M, Cummings SR, Nalls MA, Newman AB, Park V, Sanders JL, Tranah GJ, Tishkoff SA, et al. Comparison between southern blots and $\mathrm{qPCR}$ analysis of leukocyte telomere length in the health ABC study. J Gerontol A Biol Sci Med Sci. 2014;69:527-31.

14. Martin-Ruiz CM, Baird D, Roger L, Boukamp P, Krunic D, Cawthon R, Dokter MM, van der Harst P, Bekaert S, de Meyer T, et al. Reproducibility of telomere length assessment: an international collaborative study. Int J Epidemiol. 2015;44:1673-83.

15. Mefford HC, Trask BJ. The complex structure and dynamic evolution of human subtelomeres. Nat Rev Genet. 2002;3:91-102.

16. Vera E, Blasco MA. Beyond average: potential for measurement of short telomeres. Aging (Albany NY). 2012;4:379-92.

17. Biron-Shental T, Sukenik Halevy R, Goldberg-Bittman L, Kidron D, Fejgin $\mathrm{MD}$, Amiel A. Telomeres are shorter in placental trophoblasts of pregnancies complicated with intrauterine growth restriction (IUGR). Early Hum Dev. 2010:86:451-6.

18. Toutain J, Prochazkova-Carlotti M, Cappellen D, Jarne A, Chevret E, Ferrer J, Idrissi Y, Pelluard F, Carles D, Maugey-Laulon B, et al. Reduced placental telomere length during pregnancies complicated by intrauterine growth restriction. PLOS ONE. 2013;8:e54013.

19. Kirley K, Shalowitz M. Early growth patterns associated with cardiovascular disease. Curr Cardiovasc Risk Rep. 2013:7:147-53.

20. Barker DJ. Adult consequences of fetal growth restriction. Clin Obstet Gynecol. 2006;49:270-83.

21. Menon R, Yu J, Basanta-Henry P, Brou L, Berga SL, Fortunato SJ, Taylor RN Short fetal leukocyte telomere length and preterm prelabor rupture of the membranes. PLoS ONE. 2012;7:e31136.

22. Xu J, Ye J, Wu Y, Zhang H, Luo Q, Han C, Ye X, Wang H, He J, Huang H, et al. Reduced fetal telomere length in gestational diabetes. PLoS ONE. 2014;9:e86161.

23. Mathew V, Ayyar SV. Developmental origins of adult diseases. Indian J Endocrinol Metab. 2012;16:532-41.

24. Moreno-Palomo J, Creus A, Marcos R, Hernandez A. Genomic instability in newborn with short telomeres. PLoS ONE. 2014;9:e91753.

25. Tellechea M, Gianotti TF, Alvarinas J, Gonzalez CD, Sookoian S, Pirola CJ. Telomere length in the two extremes of abnormal fetal growth and the programming effect of maternal arterial hypertension. Sci Rep. 2015;5:7869.

26. de Zegher F, Diaz M, Lopez-Bermejo A, Ibanez L. Recognition of a sequence: more growth before birth, longer telomeres at birth, more lean mass after birth. Pediatr Obes. 2016;12:274-9.

27. Wojcicki JM, Olveda R, Heyman MB, Elwan D, Lin J, Blackburn E, Epel E. Cord blood telomere length in Latino infants: relation with maternal education and infant sex. J Perinatol. 2016;36:235-41.

28. Drury SS, Esteves K, Hatch V, Woodbury M, Borne S, Adamski A, Theall KP. Setting the trajectory: racial disparities in newborn telomere length. J Pediatr. 2015;166:1181-6.

29. Strohmaier J, van Dongen J, Willemsen G, Nyholt DR, Zhu G, Codd V, Novakovic B, Hansell N, Wright MJ, Rietschel L, et al. Low birth weight in $\mathrm{MZ}$ twins discordant for birth weight is associated with shorter telomere length and lower IQ, but not anxiety/depression in later life. Twin Res Hum Genet. 2015;18:198-209. 